\title{
Editorial
}

\section{Voluntad anticipada versus distanasia en la atención del adulto mayor con COVID-19 y enfermedad renal}

\author{
Advance voluntary directive versus distanasia in the care of the elderly
} with COVID-19 and kidney disease

\author{
${ }^{-}$Roberto Ramírez Marmolejo ${ }^{1,2},{ }^{\oplus}$ Leidy Yoana Aristizábal Gómez ${ }^{3,4},{ }^{\oplus}$ Laura María Gómez Franco ${ }^{5}$, \\ ${ }^{\oplus}$ Eliana Patricia Dueñas Suarez ${ }^{6},{ }^{\oplus}$ Sofía Ramírez Isaza ${ }^{7}{ }^{\oplus}$ María Paula Soto Carvajal ${ }^{7}$ \\ 'Junta Directiva, Asociación Colombiana de Nefrología e hipertensión arterial, Bogotá D.C., Colombia \\ 'Especialización en Medicina Interna, Facultad de Salud, Universidad Santiago de Cali, Cali, Colombia \\ ${ }^{3}$ Junta Directiva, Asociación Colombiana de Geriatría y gerontología, Bogotá D.C., Colombia. \\ ${ }^{4}$ Especialización en Medicina Interna, Universidad Libre, Cali, Colombia.

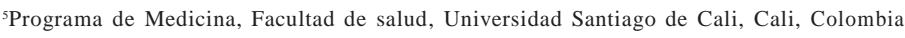 \\ ${ }^{6}$ Especialización en Geriatría, Facultad de Salud, Universidad del Valle, Cali, Colombia. \\ ${ }^{7}$ Área de ciencias, Aspaen Colombia, Cali. Colombia.
}

\section{Introducción}

$\mathrm{L}$ a presente editorial es un trabajo mancomunado del grupo interdisciplinario del Comité del Adulto Mayor de la Asociación Colombiana de Nefrología e Hipertensión Arterial, en la cual se consignan los puntos de vista de especialistas en geriatría, médicos, estudiantes y nefrólogos, y que con base en la literatura pretende hacer una reflexión sobre el alcance terapéutico más adecuado que se debe brindar a un paciente adulto mayor que tenga enfermedad renal crónica (ERC) y se infecte con COVID-19, o a un adulto mayor frágil que se infecte con COVID-19 y presente como complicación una lesión renal aguda (LRA). Todo esto encaminado a resolver algunos interrogantes como ¿Hasta dónde deben ir nuestros esfuerzos como clínicos, qué beneficio real tiene para el paciente este tipo de esfuerzos? ¿qué expectativa tiene el paciente de cómo ser atendido en sus últimos momentos de vida?

\section{Adulto mayor frágil}

La fragilidad, Según Morley et al. ${ }^{1}$, se define como un síndrome médico con múltiples causas y contribuyentes que se caracteriza por disminución de la fuerza, la resistencia y la función fisiológica, lo que incrementa la vulnerabilidad individual para desarrollar dependencia o muerte cuando se expone a un estresor. La fragilidad es altamente prevalente y puede ser física, psicológica y/o social, además tiende a ser crónica y dinámica, pudiendo progresar o ser revertida ${ }^{2}$.

Este síndrome es diferente del envejecimiento, la discapacidad y la comorbilidad; sin embargo, estos son conceptos que guardan una estrecha relación. La fragilidad se presenta sin comorbilidad o discapacidad en cerca del 26,6 \% de los casos, aunque la presentación conjunta de estas condiciones es más frecuente conforme aumenta el grado de fragilidad $^{3}$.

Varios estudios ${ }^{4-6}$ prospectivos muestran cómo la fragilidad aumenta en promedio un $60 \%$ el riesgo de hospitalización y deterioro cognitivo, y en un $80 \%$ el riesgo de pérdida de capacidad para realizar actividades de la vida diaria. Además, es un síndrome que puede duplicar el riesgo de mortalidad, limitación física, institucionalización y caídas. Es por este gran impacto de la fragilidad en el adulto mayor que

Citación: Ramírez Marmolejo R, Aristizábal Gómez LY, Gómez Franco LM, Dueñas Suarez EP, Ramírez Isaza S, Soto Carvajal MP. Voluntad anticipada versus distanasia en la atención del adulto mayor con COVID-19 y enfermedad renal. Rev. Colomb. Nefrol.2020;7(2):17-29. https://doi.org/10.22265/acnef.7.2.519

Correspondencia: Roberto Ramírez Marmolejo, robertoramire5@ hotmail.com 
numerosas especialidades han adoptado este concepto y han estudiado cómo influye en los resulta dos adversos ante diferentes intervenciones o tratamientos. De esta forma, en varias especialidades se ha encontrado una evidencia robusta entre fragilidad y resultados negativos como: mortalidad a corto o mediano plazo, complicaciones o reducción de la calidad de vida ${ }^{7}$.

En nefrología, por ejemplo, se ha observado una relación bidireccional entre fragilidad y función renal: por un lado, el deterioro en la función renal incrementa el riesgo de fragilidad, pues, en adultos mayores con ERC grado 3 se duplica la prevalencia de fragilidad en comparación con pacientes con función renal normal ${ }^{8}$, y en pacientes con diálisis la prevalencia de fragilidad puede llegar a ser del 50-70 \%; y, por el otro, los pacientes frágiles en diálisis tienen el doble de riesgos de hospitalización, institucionalización y muerte al año en comparación con pacientes no frágiles ${ }^{10,11}$, e inclusive en ERC se ha considerado la fragilidad moderada a severa como un mejor predictor de sobrevida en comparación con el grado de comorbilidad ${ }^{12}$.

El concepto de calidad de vida del adulto mayor se debe tener en cuenta en todas las esferas, incluyendo la sexual ${ }^{13}$.

Por tanto, comprendiendo la importancia de evaluar la fragilidad, se debe definir ahora cómo evaluarla. Desde la geriatría existen dos enfoques que evalúan la fragilidad de forma diferente: el fenotipo de fragilidad y la acumulación de déficits. El primero ve la fragilidad como un síndrome biológico dado por cambios biológicos relacionados con la edad que generan las características físicas específicas y, finalmente, resultados adversos, mientras que la segunda la ve como un estado de riesgos multidimensional, y además tiene en cuenta aspectos médicos, funcionales y sociales, y considera que una alta acumulación de déficits representa un envejecimiento acelerado $^{14}$. Cada uno de estos enfoques tiene varios instrumentos de tamizaje validados en atención primaria: entre los dos suman más de $27^{15}$. La herramienta de tamizaje a escoger puede variar de acuerdo a dos elementos: primero las características de la población estudiada y segundo los objeti- vos de valoración específicos, es decir en relación a la predicción de riesgos de mortalidad, complicaciones post quirúrgicas o riesgos de discapacidad ${ }^{16}$.

En el actual contexto hospitalario de la pandemia por COVID-1917, la escala clínica de fragilidad (Clinical Frail Scale- CFS) ${ }^{18}$, que hace parte del enfoque de acumulación de déficit, ha sido la más utilizada $^{19,20}$. Esta les permite a los clínicos estratificar a los adultos mayores en diferentes grados de vulnerabilidad (1 a 9), en donde un puntaje de 1 representa pacientes en muy buena forma; 2 , pacientes en forma; 3 , pacientes en buen estado; 4, pacientes vulnerables; 5 , pacientes levemente frágiles; 6 , pacientes moderadamente frágiles; 7 , pacientes con fragilidad grave; 8 , pacientes con fragilidad muy grave, y 9 , pacientes en fase terminal de una enfermedad.

La evaluación de la CFS debe basarse en el estado basal del paciente, es decir dos semanas previas al ingreso. Esta escala predice la mortalidad intra hospitalaria, independiente de la edad y el género, y se ha validado en adultos mayores en contextos de urgencias ${ }^{21}$ y en pacientes críticos ${ }^{22}$. Además, puede ser aplicada de forma fácil y rápida por el personal médico y tiene poca variabilidad en los resultados entre observadores ${ }^{23,24}$.

Es recomendable hacer una determinación temprana del estado de fragilidad, preferiblemente en el triage de los servicios de urgencias o durante las evaluaciones de seguimiento de los pacientes en consultas ambulatorias, con el objetivo de determinar la complejidad y el grado de riesgo de complicaciones y mortalidad intrahospitalaria y, de esta forma, identificar quiénes pueden beneficiarse de una valoración geriátrica integral ${ }^{25}$. Esta evaluación puede ser útil en la toma de decisiones y en la priorización de recursos, especialmente cuando se prevé el colapso de los servicios de salud como ha sucedido durante la pandemia por COVID-19.

La predicción de mortalidad en pacientes adultos mayores de acuerdo a la CFS puede variar según la patología de base, especialmente en el contexto de enfermedad moderada a grave por COVID-19. En estudios previos a la pandemia, la mortalidad intrahospitalaria de los pacientes ingresados a sala 
general con CFS 1-4 fue del $0 \%$; con CFS 5, 0-3,4 \%; con CFS $6,6,5 \%$, y con CFS 7 , de hasta $27,4 \%$. Además, entre puntajes CFS 6 a 8 se ha descrito que en la post hospitalización se presenta una mortalidad anual de 41,3\% $\%^{26-28}$. De forma paralela, la mortalidad en pacientes más críticos con criterios de ingreso a unidad de cuidados intensivos (UCI) con puntajes CFS 5 es de $20 \%$ intrahospitalaria y $50 \%$ al año y con CFS 6 a 7 es de $65 \%$ al año ${ }^{29}$.

Los estudios que han evaluado el efecto de la fragilidad en el pronóstico de los adultos mayores con COVID-19 presentan resultados variables. Chong et $a l .{ }^{30}$ mostraron que la mortalidad intrahospitalaria puede ir desde $0 \%$ entre pacientes con CFS 1-5, pasando por $4,7 \%$ en CFS 6 , hasta $8,6 \%$ en CFS 78 , y que la mortalidad al año es de $0 \%, 8,3 \%, 29,2 \%$ y $60 \%$ para CFS $1-4,5,6$ y $7-8$, respectivamente. Por su parte, Owen et al. ${ }^{31}$ encontraron que la mortalidad a 30 días en pacientes negativos para COVID19 se describió en 8,9\% para pacientes con CFS 1-3, en $24,2 \%$ para CFS $4-5$, en $26 \%$ para CFS 6 y en $32,8 \%$ para CFS 7-8, y que en pacientes con COVID19 confirmado la mortalidad fue de $61,1 \%, 62,4 \%$, $54,2 \%$ y $58,8 \%$ respectivamente, lo que evidenció muy poca correlación entre mortalidad y fragilidad ante la presencia del virus ${ }^{28}$. Finalmente, Hewit et al. ${ }^{32}$ evidenciaron que los adultos mayores con COVID-19 tuvieron una mortalidad intrahospitalaria de $7,7 \%$ en CFS1, de $11,2 \%$ en CFS2, de $19,2 \%$ en CFS 3, de 28,1\% en CFS 4, de 27,5\% en CFS 5, de $33,5 \%$ en CFS6, de $36,9 \%$ en CFS7, de $55,7 \%$ en CFS 8 y de $44,4 \%$ en CFS9. Allí, comparados con CFS 1-2, el OR de mortalidad a los 7 días fue de 1,22 (IC95\%: 0,64-2,38) para CFS 3-4, de 1,62 (IC95\%: 0,81-3,26) para CFS 5-6 y de 3,12 (IC95\%: 1,56-6,24) para CFS 7-9, lo que demostró un incremento de riesgo de mortalidad solo para este último grupo en el contexto intrahospitalario, aunque no se realizaron subanálisis en relación a la severidad de la enfermedad o el tipo de complicación ${ }^{32}$.

Estos datos orientan sobre la necesidad de evaluar la severidad de la enfermedad del paciente como otro aspecto pronóstico y sugieren que, si bien se habla de fragilidad en un rango de CFS 5-9, posiblemente los riesgos de complicaciones y la mortalidad determinen adecuaciones en los planes terapéuticos solo a partir CFS 6 en quienes ya se presenta dependencia en actividades básicas de la vida diaria.

De esta forma, en la valoración de los pacientes adultos mayores y en el planteamiento de los manejos más adecuados para este grupo de población, además del grado de fragilidad, se deben considerar otras variables que están asociadas con los resultados clínicos, tales como el tipo y severidad de la enfermedad crónica/aguda, la vulnerabilidad social, el estado nutricional y cognitivo y la presencia de otros síndromes geriátricos. Por ejemplo, los pacientes en hemodiálisis con trastorno neurocognitivo mayor tienen una probabilidad de sobrevida del $30 \%$ a dos años versus $60 \%$ de aquellos sin trastorno cognitivo, además de una reducción del $50 \%$ de su expectativa de vida ${ }^{11}$.

De igual forma, para respetar los principios éticos de beneficencia, no maleficencia, preservación de la autonomía y justicia distributiva, los médicos deben actuar defendiendo los intereses de los pacientes de manera transparente para maximizar la toma de decisiones autónoma y no causar daño ${ }^{33}$. Así, las variables de laboratorio o la edad por sí mismas y en forma aislada no deben orientar las conductas sobre los adultos mayores. De esta forma, como parte de un manejo centrado en la persona, en la evaluación del riesgo de ciertas intervenciones (incluyendo la diálisis) y en la toma de decisiones, se deberían incluir al menos cuatro elementos claves: la severidad de la enfermedad, la probabilidad que las intervenciones médicas sean exitosas o beneficiosas, el grado de fragilidad, su funcionalidad $\mathrm{y}$, desde luego, los deseos del paciente y su familia o cuidadores $^{34}$.

\section{Distanasia - obstinación terapéutica}

A medida que la medicina ha avanzado, a lo largo de la historia se han dado avances en el control de las enfermedades y, por tanto, la expectativa de vida de la población se ha incrementado. El resultado actual es que nos encontramos con pacientes con múltiples comorbilidades, en edades avanzadas, extremadamente frágiles y que por un motivo u otro presentan daño renal agudo y en quienes se debe 
tomar la decisión de iniciar o no una terapia de reemplazo renal.

La distanasia se define, en pocas palabras, como la no prolongación de la vida sino del sufrimiento ${ }^{35}$. También conocida como obstinación terapéutica, es aquella práctica clínica donde, sin beneficio real de la salud del paciente ni de su calidad de vida, se prolonga de manera artificial el proceso de morir, lo que causa mayor deterioro funcional, mayor morbilidad por las intervenciones $y$, finalmente, mayor sufrimiento.

Es claro que, para el pensamiento humano, la muerte genera mucho rechazo; esto es reforzado con el pensamiento mágico de la comunidad occidental donde uno de los finales escatológicos que podemos tener es el infierno. En este sentido, es normal que el personal de salud luche en contra de la muerte de manera frontal y que los familiares del paciente con multimorbilidades que se infecte con COVID-19 y presente LRA exijan resultados que en muchas ocasiones van en contra de las limitadas capacidades del personal de salud.

El enfoque de iniciar o no terapia de reemplazo renal en un paciente con un cuadro de LRA por COVID$19^{36}$ debe ir más allá de la simple discusión sobre si existe o no el recurso tecnológico (para motivos de discusión en este paper, se asume que sí se cuenta con la disponibilidad de cualquiera de las terapias de reemplazo renal para la atención del paciente con LRA secundaria a infección por COVID-19).

Vincent $\frac{37}{3}$, en 1999, publicó un estudio en el que mostró que el $73 \%$ de los intensivistas recibían en la UCI pacientes sin pronóstico de vida, un panorama que claramente ha cambiado en las últimas décadas, pero que sigue presentándose en la cotidianidad. Asimismo, un factor que influye cada vez más en esta situación es la presión de la familia para que se le apliquen todos los recursos posibles al paciente, a pesar de que él previamente y en estado de clara lucidez haya manifestado su deseo de no recibir medidas terapéuticas extremas ni invasivas.

Esta presión de la familia pone en una fuerte encrucijada al clínico, en especial cuando se le ame- naza con demandas o incluso se le agrede verbal o físicamente, obligándolo en algunas circunstancias a iniciar o persistir medidas terapéuticas distanásicas. Esto es aún más grave cuando se usan los medios de comunicación masivos, como la televisión o las redes sociales, para hacer presión; allí, sin poseer conocimientos científicos y a pesar de haber discutido y entendido la situación con los médicos tratantes, se tergiversa la información y se difunden datos falsos o incorrectos de tal manera que se afecta el buen nombre de los profesionales tratantes.

Con base en lo anterior, se crea el término "medicina defensiva", el cual Monteiro ${ }^{38}$ define como la decisión o acción clínica que toman los médicos para protegerse de la acusación de una mala práctica médica, lo cual es lamentable pero cada vez más frecuente.

\section{Principio de no maleficencia "primum non nocere"}

Es uno de los cuatro principios que rige la bioética moderna. En muchas ocasiones, los médicos, de manera involuntaria, podemos incurrir en su trasgresión al aplicar la distanasia (como iniciar terapias de reemplazo renal cuando el pronóstico del paciente es completamente reservado) dado que podemos llevar a un paciente a medidas extremas e innecesarias que son potencialmente prolongadoras de agonía y dolor, deterioran la calidad de vida y entorpecen el proceso de muerte digna. En otros casos, hay pacientes que pueden pedir maleficencia para ellos, solicitando no una decisión anticipada de no reanimar o de no tomar medidas extremas, sino una muerte anticipada (eutanasia activa), en donde lo que se puede observar después de una evaluación multidisciplinaria es que el paciente realmente padece de una depresión severa y lo que en realidad tiene es ideación suicida, lo cual va en total contracorriente con el concepto de voluntad anticipada, donde el paciente debe demostrar un pensamiento con total autonomía y libre de patología psiquiátrica o cognitiva.

La voluntad anticipada es una forma de garantizar a un paciente en estado no terminal que, en 
caso de pronóstico reservado, se respetará su decisión de no ofrecerle medidas extremas o indeseadas para el concepto cosmológico del propio paciente y que el hacerlo o dejarlo de hacer no afectará su pronóstico final, sino que se le brindará una muerte digna e idealmente en compañía de sus seres queridos.

Respecto a la decisión médica, Azulay-Tapiero ${ }^{39}$ afirma que

"La actitud que debe tomar ante los dilemas que aparecen no debe ser producto de la intuición ni de su estado de ánimo en ese momento, sino que debe estar basada en una reflexión prudente, informada, discutida y compartida por el resto de los miembros del equipo interdisciplinar $\mathrm{y}$, a ser posible, conociendo y teniendo muy en cuenta cuáles son las preferencias del enfermo" 39 .

\section{¿Qué opinan los pacientes adultos mayores?}

A continuación se sintetizan algunos testimonios de adultos mayores o sus familiares sobre su opinión acerca de la distanasia y/o sobre la voluntad anticipada.

\section{Mujer de 78 años con enfermedad cerebral isquémica}

Una paciente que a causa de una enfermedad cerebro vascular (ECV) isquémica se encontraba postrada en cama e indicó que quería "irse" de manera natural y no alargar su enfermedad con una forma de vida que no podía disfrutar: Ella comentó que su padre había fallecido 5 años atrás después de una larga agonía, que durante el tiempo que él estuvo enfermo ella sufrió al verlo padecer con los tratamientos y deteriorarse sin signos de mejoría y que, por tanto, no quería que sus hijos sufrieran de la misma forma. La mujer no deseaba seguir un tratamiento que solo le alargaría la fecha de su muerte, por lo que su familia le cumplió su deseo y murió en casa bajo sedación. Su hijo aseguró que "se fue en paz" ${ }^{40}$.

\section{Mujer de 82 años con trauma craneoencefálico severo}

Después de una dura caída, la paciente sufrió una hemorragia cerebral y como consecuencia no podía comer ni hablar y sufría de constantes convulsiones que no solo la afectaban a ella, sino también a sus familiares quienes se sentían impotentes de no poder hacer nada por ella. Al ser su trauma severo, la única forma de ayudarla era cumpliendo sus deseos de poder morir en paz, pues los médicos indicaron que se encontraba en un coma irreversible y que no se podía demostrar si sufría. Su familia quería calmar el dolor, además estando consciente le había dicho muchas veces a su hija que ella no quería sentir cómo agonizaba y que solo quería descansar, para lo cual usó frases como "los paliativos quitan el dolor, pero no la conciencia de la propia muerte" y "no quiero verme nunca en una situación así". Luego de 20 días de la caída, fue trasladada a la casa de su hija, donde murió de una forma tranquila en el calor de su hogar ${ }^{41}$.

\section{Mujer de 69 años con esclerosis múltiple}

Paciente con diagnóstico de una esclerosis múltiple que se convirtió en una constante agonía sin fin por su progresivo deterioro neurológico. La mujer pasó a ser una persona dependiente que diariamente necesitaba de la ayuda de los demás para poder hacer simples actividades cotidianas como comer o ir al baño, lo que la hacía sentirse inferior. Después de mucho tiempo de sufrimiento decidió firmar su voluntad de morir, pues quería descansar y morir de una forma tranquila. Totalmente consciente de su decisión, tuvo que pedirle ayuda a su esposo para firmar la constancia ya que era incapaz de mover las manos, aunque gozaba de sus facultades intelectuales y conciencia a plenitud ${ }^{42}$.

\section{Hombre de 79 años con cáncer oral}

Paciente que padeció cáncer oral durante cinco años, el cual le comprometió el rostro, le causaba un dolor bucal constante y le generaba dificultad para ingerir alimentos. El médico tratante dijo que, aunque su cáncer no tenía cura y que era una persona totalmente dependiente, era "un paciente funcional 
y que la enfermedad no supone su muerte inmediata". El sujeto en varias ocasiones buscó amparo del derecho de morir con dignidad y en una entrevista con una radio local reiteró su deseo de que se le practicara el procedimiento de muerte anticipada: "Quiero morir en mis cinco sentidos y no esperar a que este mal me deje hecho una miseria en medio de este dolor insoportable". Además, él decía "no dejes para mañana lo que puedes hacer hoy"43, refiriéndose a terminar con el sufrimiento mediante eutanasia activa.

\section{Mujer de 66 años con poliomielitis}

Cuando tenía 2 años de edad, esta paciente fue diagnosticada con poliomielitis y desde entonces sufrió diversas complicaciones de salud, siendo la columna el órganos más afectado, por lo que tuvo que usar silla de ruedas para movilizarse durante 52 años, pero a pesar de todas las adversidades ella trabajaba y luchaba por su sustento y el de su hermana y su sobrina; sin embargo, una caída la dejó postrada en cama de por vida. La sumatoria de todas sus complicaciones la llevó a tomar la decisión de firmar su constancia de voluntad anticipada, en la cual se niega a recibir ayudas artificiales para prolongar su vida innecesariamente. A ella le gustaría permanecer esta última etapa de su vida en su casa debido a que su enfermedad no le permite realizar ningún tipo de actividad y solo quiere estar junto a su familia y evitar más sufrimiento ${ }^{44}$.

\section{Mujer de 73 años con esclerosis lateral amiotrófica}

Paciente totalmente dependiente del cuidador que comenzó a caerse continuamente, e incluso se fracturó el peroné, además le picaba la piel, se le deformó el dedo índice de la mano izquierda, comenzó a fatigarse, a hablar poco y a cansarse con mayor frecuencia, tampoco podía reírse. Antes de la enfermedad, ella era una mujer muy activa y autosuficiente que realizaba muchas actividades familiares, pero en el año 2000 luego de recibir el diagnóstico de esclerosis lateral amiotrófica la familia debió empezar a ayudarle con actividades cotidianas como levantarla, vestirla, bañarla y suministrarle los alimentos, no podía realizar actividades por sus propios medios y te- nía muchas complicaciones y dolores. Lo único que quería era "liberar a la familia de cargas posteriores" ${ }^{45}$, y liberarse de su propio sufrimiento.

\section{Derechos del adulto mayor}

Los derechos del adulto mayor hoy en día están regidos mundialmente por los Principios de las Naciones Unidas en favor de las Personas de Edad ${ }^{46}$, donde se establecen cinco derechos fundamentales: a la independencia, a la participación, a los cuidados, a la autorrealización y a la dignidad. En Colombia, al ser uno de los países miembros de la ONU por constitución internacional, el Estado debe garantizar el cumplimiento de estos derechos en su territorio nacional. En este sentido, es importante profundizar acerca del derecho al cuidado y el derecho a la dignidad, los cuales tiene como pilar el respeto de la autonomía y bienestar de las personas mayores.

El derecho al cuidado hace énfasis en el cuidado y la protección que deben recibir los adultos mayores por parte de la familia y la comunidad donde residan y se refiere a que deben gozar de fácil acceso a los servicios de salud con el fin de recuperar o mantener un nivel óptimo de bienestar en todos los ámbitos de su vida (físico, psicológico y social) para prevenir o detener la progresión de enfermedades; también deben de tener un fácil acceso a servicios sociales y jurídicos para velar por sus derechos de autonomía, protección y cuidado, y promover que sus derechos no se vulneren mientras residan en hogares o instituciones que les brinden cuidados o tratamiento, de tal manera que allí prime su dignidad, creencias, necesidades e intimidad, así como su derecho a adoptar decisiones sobre su cuidado y su calidad de vida.

El derecho a la dignidad hace referencia a una vida digna, segura y libre de explotaciones y malos tratos físicos o mentales que debe de tener un anciano, esto a través de un trato respetuoso sin distinción de edad, sexo, raza, étnica, discapacidad, situación económica u otras ${ }^{46}$.

Del mismo modo, en junio del 2015 fue aprobada por la Asamblea General de la Organización de Es- 
tados Americanos la Convención interamericana sobre la protección de los derechos humanos de las personas mayores realizada por la Organización Panamericana de la Salud (OPS) y la Organización de Estados Americanos (OEA). Argentina, Brasil, Chile, Bolivia, Costa Rica y Uruguay fueron los primeros países en firmar; y estos últimos cuatro ya la han ratificado. De esta forma, la Comisión Económica para Latinoamérica y el Caribe (CEPAL), la OEA y la OPS han hecho un llamado a los demás países de la región para que adopten estas medidas que tienen como fin promover y proteger los derechos de las personas mayores y satisfacer sus necesidades. Los derechos fundamentales que se mencionan en esta convención son los siguientes ${ }^{47}$ :

- A la igualdad y no discriminación por razones de edad.

- A la vida y la dignidad en la vejez.

- A la independencia y la autonomía.

- A la participación e integración comunitaria.

- A la seguridad y a una vida sin ningún tipo de violencia.

- A no ser sometido a tortura ni a penas o tratos crueles, inhumanos o degradantes.

- A brindar consentimiento libre e informado en el ámbito de la salud.

- A la libertad personal.

- A la libertad de expresión y de opinión y al acceso a la información.

- A la nacionalidad y a la libertad de circulación.

- A la privación y a la intimidad.

- A la seguridad social.

- Al trabajo.

- A la salud.

- A la educación.

- A la cultura.

- A la recreación, el esparcimiento y el deporte.

- A la vivienda.

- A la propiedad.

- A un medio ambiente sano.

- A la accesibilidad y a la movilidad personal.

- A ejercer sus derechos políticos.

- A igual reconocimiento como persona ante la ley.

- $\mathrm{Al}$ acceso a la justicia.

- A poder reunirse e integrarse con familiares, amigos y/o conocidos.
De estos derechos se resaltan los siguientes:

El derecho de la vida y la dignidad en la vejez, que habla acerca de las medidas que se deben adoptar para garantizar a las personas mayores el goce efectivo de una vida digna en la vejez hasta el fin de sus días en igualdad de condiciones que cualquier otra persona que se encuentre en una etapa de vida distinta. De esta forma, los Estados deben velar por un acceso no discriminatorio a cuidados integrales de las instituciones públicas y privadas; garantizar el manejo apropiado de los problemas relacionados con el miedo a la muerte de los enfermos terminales y el dolor crónico; evitar el aislamiento, el sufrimiento y las intervenciones innecesarias, y hacer valer el derecho de la persona mayor a expresar su consentimiento informado y tomar sus propias decisiones de manera independiente y libre de presiones externas.

El derecho a la independencia y a la autonomía, que reconoce el derecho de la persona mayor de definir su plan de vida de forma libre e independiente de acuerdo a sus tradiciones y creencias y de contar con herramientas que le permitan ejercer sus derechos. En este sentido, los Estados deben adoptar programas, políticas o acciones para facilitar y promover el pleno goce de estos derechos por las personas mayores, así como favorecer su autorrealización, el fortalecimiento de las familias y de su vida en sociedad, y si es el caso también de sus relaciones afectivas. De igual forma se deberá garantizar el respeto a la autonomía de la persona mayor en la toma de sus decisiones, así como su independencia en la realización de sus actos; que las personas mayores tengan la oportunidad de elegir dónde y con quién vivir (en igualdad de condiciones que los demás) y no se vean obligadas a vivir con arreglo a un sistema de vida específico, y que la persona mayor tenga acceso progresivamente a una variedad de servicios de asistencia domiciliaria y residencial, así como a otros servicios de apoyo de la comunidad, incluida la asistencia personal, que sean necesarios para facilitar su existencia, y para evitar su aislamiento.

En el caso de Colombia, la Ley 23 de $1981^{48}$ que habla sobre los principios de la ética médica cita lo siguiente: "El médico respetará la libertad del enfermo para prescindir de sus servicios". De este 
modo, al ser el paciente adulto mayor consciente de sus actos y decisiones, el médico tratante debe respetar la decisión que este tome, sobre su salud, el tipo de tratamiento que desea y la disposición de si se le realizan o no posibles intervenciones no previstas que se requieran durante el transcurso de su enfermedad, respetando así el acuerdo de voluntad en la relación médico-paciente que se establece antes o durante la atención.

Adicionalmente, en la Ley 1251 de $2008^{49}$ se establece que se debe "proteger, promover, restablecer y defender los derechos de los adultos mayores", por lo que el Estado, la sociedad y la familia tienen como deber diseñar políticas, planes y programas para regular el funcionamiento y cumplimiento por parte de las instituciones prestadoras de servicio en la promoción de una atención integral a las personas en etapa de la vejez, teniendo siempre como principio el derecho a la autonomía del paciente adulto mayor $\mathrm{y}$, respetando las decisiones que este tome con respecto a la evolución de los tratamientos y los procedimientos necesarios en el curso de su enfermedad.

\section{Voluntad anticipada}

La planificación anticipada de la atención es vital en el contexto actual de la pandemia por COVID19 debido al rápido e impredecible deterioro que se observa en algunos pacientes, principalmente en la población adulta; este es un problema particularmente urgente para las personas con trastorno neurocognitivo mayor que pueden carecer de la capacidad para tomar estas decisiones por sí mismas ${ }^{33}$. De acuerdo a los principios éticos que deben regir el actuar médico, el principio de autonomía es un concepto introducido por Kant en la ética y que etimológicamente significa la capacidad de darse a uno mismo las leyes; en bioética tiene un sentido más concreto y se identifica con la capacidad de tomar decisiones y de gestionar el propio cuerpo y, por lo tanto, la vida y la muerte de los seres humanos ${ }^{50}$. Este concepto se nutre de diversas disciplinas que se interesan por el respeto del ser humano, de sus derechos y de su dignidad y en este contexto la autonomía es una expresión de la dignidad de los seres humanos ${ }^{51}$.
En Colombia este derecho puede ejercerse a través del documento de voluntad anticipada (DVA) que promulga la Resolución 2665 de $2018^{52}$, la cual lo define como el documento en el que "toda persona capaz, sana o en estado de enfermedad, en pleno uso de sus facultades legales y mentales y como previsión de no poder tomar decisiones en el futuro, declara, de forma libre, consciente e informada su voluntad sobre las preferencias al final de la vida que sean relevantes para su marco de valores personales".

El DVA garantiza el derecho al libre desarrollo de la personalidad y a la autonomía de la persona que lo suscriba y que posteriormente se encuentre, por diversas circunstancias y en determinado momento, en imposibilidad de manifestar su voluntad. En todo caso, no suplanta la posibilidad de decidir y expresar su consentimiento actual en el proceso de atención si la persona es capaz y está en pleno uso de sus facultades legales y mentales.

Además, si bien la resolución indica que el DVA debe estar por escrito, también señala que son admisibles las declaraciones de voluntad anticipada expresadas en videos o audios y otros medios tecnológicos. Este documento debe incluir como mínimo la siguiente información:

- Ciudad y fecha de expedición del documento.

- Nombres, apellidos y número de documento de identificación de la persona que desea manifestar su voluntad anticipada.

- Indicación concreta y específica de que se encuentra en pleno uso de sus facultades mentales y libre de toda coacción y que conoce y está informado de las implicaciones de su declaración.

- Manifestación específica, clara, expresa e inequívoca respecto a sus preferencias en relación al cuidado futuro de su salud e integridad física, así como indicaciones concretas de su cuidado y las preferencias para el final de la vida que considere relevantes en el marco de sus valores personales, su entorno cultural, sus creencias religiosas o su ideología.

- Firma de la persona declarante.

El DVA debe formalizarse, y puede hacerse ante un notario a través de escritura pública, ante dos

24 Voluntad anticipada versus distanasia en la atención del adulto mayor con COVID-19

y enfermedad renal

e2500-5006 Revista Colombiana de Nefrología 
testigos o ante el médico tratante. El paciente se beneficiará de estas voluntades garantizándose la posibilidad de elegir de manera anticipada sobre los eventos que pueden afectar su salud, su integridad personal o su vida. En este orden de ideas, el DVA es un instrumento que busca proteger la autonomía del paciente, evita la extralimitación terapéutica y favorece el inicio oportuno del cuidado paliativo ${ }^{53}$.

Sánchez-Tomero et al..$^{54}$, antes del inicio de la pandemia por COVID-10, publicaron los resultados de una encuesta realizada a 265 pacientes y contestada por 154 (69,2\% respondió sin ayuda), mediante la cual encontraron que el grado de implantación del DVA y de designación de representante era muy bajo $(7,9 \%$ y $6,6 \%$, respectivamente). En dicho estudio los entrevistados tenían una idea muy clara sobre sus deseos ante situaciones como coma irreversible, estado vegetativo, demencia irreversible y enfermedad grave no tratable y cerca del $65 \%$ no deseaba que se les aplicara ventilación mecánica, alimentación por sonda, diálisis o maniobras de resucitación en caso de paro cardiorrespiratorio. Asimismo, muchos opinaron que el DVA podría hacerse antes de comenzar el tratamiento de diálisis, aunque la mayoría consideró que solo debería ofrecerse a aquellas personas que lo pidieran; por lo tanto, los autores concluyen que los pacientes en diálisis tienen una postura bastante definida ante diversas situaciones asociadas con el fin de la vida; sin embargo, esta voluntad no está reflejada documentalmente debido a la escasa implantación de los $\mathrm{DVA}^{54}$.

Rodríguez-Jornet et al. ${ }^{55}$ aplicaron por medio de entrevistas telefónicas a pacientes que iniciaban hemodiálisis, y en un periodo de 10 años, 443 cuestionarios que abordaban circunstancias clínicas hipotéticas que ayudan a los pacientes y sus familias en la toma de decisiones. El 41,3\% de los entrevistados manifestó que deseaba limitar los esfuerzos terapéuticos en las situaciones clínicas graves que se presentaban, el $37,9 \%$ se negó a completar el cuestionario, el 14,7 \% expresó sus deseos sin confirmación por escrito y el 6,1\% expresó su deseo de continuar en diálisis en todas las situaciones. 224 pacientes habían fallecido en la fecha de finalización del estudio., siendo la muerte la causa de retirada del programada de diálisis en el $20,2 \%$ de los caos. Los representantes informaron un grado de satisfacción extremadamente alto con el cuestionario $(94,7 \%)$. Como hallazgo destacable, los autores encontraron que las personas más jóvenes fueron más reacias a considerar y responder cuestionarios sobre directrices anticipadas ${ }^{55}$.

El DVA es de suma importancia en adultos mayores con ERC dada la evidencia disponible del impacto de esta comorbilidad. Al respecto, Ssentongo et $a l .{ }^{56}$ publicaron un metaanálisis donde relacionan la mortalidad por COVID-19 con las comorbilidades prexistentes; allí incluyeron 25 estudios que tuvieron una heterogeneidad media entre sí y un bajo sesgo de publicación con alta calidad. Las comorbilidades previas relacionadas fueron enfermedad cardiovascular (cociente de riesgos (RR): 2,25; IC95 \%: 1,60-3,17; número de estudios (n): 14), hipertensión (RR: 1,82; IC95 \%: 1,43-2,32; n: 13), diabetes (RR: 1,48; IC95 \%: 1,02-2,15; n: 16), insuficiencia cardíaca congestiva (RR: 2,03; IC95 \%: 1,28-3,21; n=3), ERC (RR: 3,25 IC95 \%: 1,13-9,28); n: 9) y cáncer (RR: 1,47; IC95 \%: $1,01-2,14 ; \mathrm{n}: 10)^{56}$.

Las personas que viven con ERC avanzada, tratadas con o sin terapia de reemplazo renal, presentan una función física auto informada más baja y un rendimiento físico deteriorado, lo que contribuye a una alta prevalencia de fragilidad, discapacidad de movilidad y mayor riesgo de mortalidad ${ }^{57}$; por lo tanto, en la toma de decisiones en el adulto mayor con ERC y COVID-19 las voluntades anticipadas tienen un rol fundamental. Por tanto, se recomienda que estas sean conocidas previo al proceso infeccioso y que el hacerlas oficiales sea una práctica común de la nefrogeriatría dado que el escenario de urgencias frente a una insuficiencia respiratoria aguda no es el más ideal para hacerlo. De este modo, la Asociación de Cuidados Paliativos de Colombia recomienda que en caso de que esta voluntad no sea conocida previamente, se suscriba en las áreas de hospitalización de COVID-19 en pacientes que se encuentren estables; sin embargo, se destaca que la mayor importancia es el diligenciamiento en pacientes con estas comorbilidades que son crónicas, irreversibles y progresivas en el tiempo ${ }^{58}$.

Según la revisión de la literatura que se realizó para el presente estudio, hasta el momento no hay 
estudios realizados en Colombia con adultos mayores con ERC en contexto de COVID-19. Por tanto, y debido a la evidencia presentada, es necesario hacer un llamado de atención a la comunidad médica, principalmente la de las unidades renales, para que informen a los pacientes sobre este derecho e inviten a suscribir el DVA, que permitirá una planificación anticipada de la atención según los deseos del paciente, no solo en el escenario de pandemia sino como una práctica médica generalizada, basada en los principios éticos y centrada en la persona.

\section{Conclusiones}

Los adultos mayores con COVID-19 que deben ser ingresados a una UCI quedan con secuelas luego de su recuperación, tales como discapacidad y delirium. A la fecha se desconoce qué tan irreversibles serían estas secuelas, pero una adecuada estimación de la edad biológica podría predecir un potencial de rehabilitación y establecer un plan de tratamiento.

Es importante dar a conocer a los pacientes que no es lo mismo voluntad anticipada y eutanasia activa o pasiva. La primera es un documento firmado por una persona de manera consciente, en su libre derecho y autonomía para decidir cómo quiere morir (nunca de manera precipitada ni sin renunciar a la atención médica), mientras que la segunda es una decisión que se toma a último momento y consiste en cortar con la vida. En cualquiera de las dos opciones, la calidad de vida y la dignidad deben ser primordiales hasta la muerte de los pacientes.

Se puede decir que el adulto mayor frágil es aquel que tiene un incremento de la vulnerabilidad física o biológica y que genera un mayor riesgo de discapacidad o muerte ante un factor estresante. Esta fragilidad se relaciona de manera directa y proporcional con la progresión de la ERC como causa-efecto de la misma: la ERC en grado 1-2 se relaciona con índices de fragilidad de 2-3 y la ERC en grado 5, con índices de fragilidad $>7$. Es importante mencionar que la fragilidad del adulto mayor con esta enfermedad está subestimada.
El diagnóstico de fragilidad debería ser activo en toda consulta de rutina de un adulto mayor, pues muchas veces pasa desapercibido y si se hace de manera temprana es posible revertir sus efectos adversos, en especial la pérdida de autonomía. Para hacer este diagnóstico se debe tener en cuenta el estado previo del paciente antes de su hospitalización para compararlo con su estado actual. Asimismo, las decisiones se deben tomar con principios éticos y de manera interdisciplinaria (con líderes espirituales, sociólogos, chamanes, etc.)

La prioridad para la atención del adulto mayor es una muerte digna, en compañía de sus seres queridos, y para esto es de vital importancia tener en cuenta la opinión autónoma del paciente y sus derechos, los cuales deben dar a conocer los profesionales de la salud. De este modo, se deben replantear los protocolos donde el adulto mayor debe permanecer solo en su hospitalización para permitir que al menos un familiar de bajo riesgo lo acompañe con las medidas de bioseguridad necesarias. De igual forma, se debe tener en cuenta el estado emocional del paciente, ya que en algunos casos su deseo de morir es consecuencia de una depresión momentánea y no una verdadera decisión autónoma.

Los medios de comunicación muchas veces han intentado tomar decisiones y/o presionar a la comunidad, desconociendo los aspectos fisiopatológicos de las enfermedades y las condiciones particulares de cada paciente. Por lo tanto, se recomienda, antes de difundir públicamente cualquier tipo de opinión, buscar asesoría tanto con el médico tratante, como con un grupo de médicos independientes que brinden una segunda opinión.

En síntesis, se deben respetar las decisiones de los pacientes, incluida el cómo quieren llegar a sus últimos días, ya que generalmente los familiares no lo hacen. De este modo, es necesario hacer una reflexión comunitaria sobre el respeto a los derechos de las personas mayores. 
Rev. Colomb. Nefrol. 2020;7(2):17-29, julio-diciembre de 2020 http://www.revistanefrologia.org

http://dx.doi.org/10.22265/acnef.7.2.519

\section{Referencias}

1. Morley JE, Vellas B, van Kan GA, Anker SD, Bauer JM, Bernabei R, et al. Frailty Consensus: A Call to Action. J Am Med Dir Assoc. 2013;14(6):392-7. https://dx.doi.org/10.1016/j.jamda.2013.03.022.

2. Dent E, Martin FC, Bergman H, Woo J, Romero-Ortuno R, Walston JD. Management of frailty: opportunities, challenges, and future directions. Lancet. 2019;394(10206):1376-86. https://dx.doi.org/10.1016/S0140-6736(19)31785-4.

3. Clegg A, Young J, Iliff S, Olde-Rikkert M, Rockwood K. Frailty in elderly people. Lancet. 2013;381(9868):752-62. https://dx.doi.org/ 10.1016/S0140-6736(12)62167-9.

4. Rockwood K, Howlett SE, MacKnight C, Beattie BL, Bergman H, Hébert R, et al. Prevalence, attributes, and outcomes of fi tness and frailty in community-dwelling older adults: report from the Canadian study of health and aging. J Gerontol A Biol Sci Med Sci. 2004;59(12):1310-17. https://dx.doi.org/10.1093/gerona/59.12.1310.

5. Bandeen-Roche K, Xue QL, Ferrucci L, Walston J, Guralnik JM, Chaves P, et al. Phenotype of frailty: characterization in the women's health and aging studies. J Gerontol A Biol Sci Med Sci. 2006;61(3):262-6. https://dx.doi.org/10.1093/gerona/61.3.262.

6. Ensrud KE, Ewing SK, Taylor BC, Fink HA, Cawthon PM, Stone KL, et al. Comparison of 2 frailty indexes for prediction of falls, disability, fractures, and death in older women. Arch Intern Med. 2008;168(4):382-89. https://dx.doi.org/10.1001/ archinternmed.2007.113.

7. Hoogendijk EO, Afilalo J, Ensrud KE, Kowal P, Onder G, Fried LP. Frailty: implications for clinical practice and public health. Lancet. 2019;394(10206):1365-75. https://dx.doi.org/10.1016/S0140-6736(19)31786-6.

8. Musso CG, Jauregui JR, Macías-Núñez JF, Covic A, editores. Clinical Nephrogeriatrics. An Evidence-Based Guide. Sprineger; 2019. https://dx.doi.org/10.1007/978-3-030-18711-8.

9. Musso CG, Jaureguie JR, Macías-Núñez JF. Frailty phenotype and chronic kidney disease: a review of the literature. Int Urol Nephrol. 2015;47(11):1801-7. https://dx.doi.org/10.1007/s11255-015-1112-z.

10. Johansen KL, Chertow GM, Jin C, Kutner NG. Significance of frailty among dialysis patients. J Am Soc Nephrol. 2007;18(11):29607. https://dx.doi.org/10.1681/ASN.2007020221.

11. Swidler M. Chapter 37: Dialysis Decisions in the Elderly Patient With Advanced CKD and the Role of Nondialytic Therapy. En: Miller M, O’Hare AM, Shim RL Geriatric Nephrology Curriculum. American Society of Nephrology; 2009.

12. Pyart R, Aggett J, Goodland A, Jones H, Prichard A, Pugh J, et al. Exploring the choices and outcomes of older patients with advanced kidney disease. PLoS ONE. 2020;15(6):e0234309. https://doi.org/10.1371/journal.pone.0234309.

13. Ramirez-Marmolejo R, Villanueva-Bendek I, Gomez-Franco LM. Recomendaciones sobre sexualidad en el paciente adulto mayor multimorbido con enfermedad renal crónica durante la pandemia por COVID-19. Rev. Colomb. Nefrol. 2020;7(Suppl 2). https:// doi.org/10.22265/acnef.7.Supl.2.421.

14. Walston J. Frailty. Burton JR, Lee A, Potter JF, editores. Geriatrics for Specialists. Springer; 2017.

15. Theou O, Walston J, Rockwood K. Operationalizing Frailty Using the Frailty Phenotype and Deficit Accumulation Approaches. Interdiscip Top Gerontol Geriatr. 2015;41:66-73. doi: https://dx.doi.org/10.1159/000381164.

16. Dent E, Morley JE, Curz-Jentoft AJ, Woodhouse L, Rodríguez-Maña L, Fried LP, et al. Physical frailty: ICFSR international clinical practice guidelines for identification and management. J Nutr Health Aging. 2019;23(9):771-87 https://dx.doi.org/10.1007/s12603019-1273-z.

17. Ramirez-Marmolejo R, Gómez-Franco LM, Ramírez-Isaza S. Recomendaciones sobre el ingreso y reingreso a diálisis crónica de pacientes provenientes de hospitalización. Rev. Colomb. Nefrol. 2020;7(Suppl 2). https://doi.org/10.22265/acnef.7.Supl.2.435.

18. Rockwood K, Song X, MacKnight C, Bergman H, Hogan DB, McDowell I, et al. A global clinical measure of fitness and frailty in elderly people. CMAJ. 2005;173(5):489-95. https://dx.doi.org/10.1503/cmaj.050051.

19. Nickel C, Ruegg M, Pargger H, Bingisser R. Age, comorbidity, frailty status: effects on disposition and resource allocation during the COVID-19 pandemic. Swiss Med Wkly. 2020;150:w20269. https://dx.doi.org/10.4414/smw.2020.20269.

20. National Institute for Health and Care Excellence (NICE). COVID-19 rapid guideline: critical care in adults. NICE; 2020 [citado octubre 7 2020]. Disponible en: https://www.nice.org.uk/guidance/ng159/resources/critical-care-admission-algorithm-pdf-8708948893. 
Rev. Colomb. Nefrol. 2020;7(2):17-29, julio-diciembre de $2020 \mathrm{http}: / /$ www.revistanefrologia.org

http://dx.doi.org/10.22265/acnef.7.2.519

21. Kaeppeli T, Rueegg M, Dreher-Hummel T, Brabrand M, Kabell-Nissen S, Carpenter CR, et al. Validation of the Clinical Frailty Scale for Prediction of Thirty-Day Mortality in the Emergency Department. Ann Emerg Med. 2020;76(3):291-300. https://doi.org/10.1016/ j.annemergmed.2020.03.028.

22. Muscedere J, Waters B, Varambally A, Bagshaw SM, Boyd JG, Maslove D, et al. The impact of frailty on intensive care unit outcomes: a systematic review and meta-analysis. Intensive Care Med. 2017;43(8):1105-22. https://dx.doi.org/10.1007/s00134-017-4867-0.

23. Elliott A, Phelps K, Regen E, Conroy SP. Identifying Frailty in the Emergency Department-feasibility. Age Ageing. 2017;46(5):8405. https://dx.doi.org/10.1093/ageing/afx089.

24. Lewis ET, Dent E, Alkhouri H, Kellett J, Williamson M, Asha S, et al. Which frailty scale for patients admitted via Emergency Department? A cohort study. Arch Gerontol Geriatr. 2019;80:104-14. https://dx.doi.org/10.1016/j.archger.2018.11.002.

25. Wallis SJ, Wall J, Birami RWS, Romero-Ortuno R. Association of the clinical frailty scale with hospital outcomes. QJM. 2015;108(12):9439. https://dx.doi.org/10.1093/qjmed/hcv066.

26. Gregorevic KJ, Hubbard RE, Lim WK, Katz B. The clinical frailty scale predicts functional decline and mortality when used by junior medical staff: a prospective cohort study. BMC Geriatrics. 2016;16:117. https://dx.doi.org/10.1186/s12877-016-0292-4.

27. Chong E, Chia JQ, Law F, Chew J, Chan M, Lim WS. Validating a standardised approach in administration of the Clinical Frailty Scale in hospitalised older adults. Ann Acad Med Singapore. 2019;48(4):115-24.

28. Basic D, Shanley C. Inpatient Population: Using the Clinical Frailty Scale to Predict Patient Outcomes. J Aging Health. 2015;27(4):67085. https://dx.doi.org/10.1177/0898264314558202.

29. Brummel NE, Bell SP, Girard TD, Pandharipande PP, Jackson JC, Morandi A, et al. Frailty and Subsequent Disability and Mortality among Patients with Critical Illness. Am J Respir Crit Care Med. 2017;196(1):64-72. https://dx.doi.org/10.1164/rccm.20160509390C.

30. Chong E, Chan M, Tan HN, Lim WS. COVID-19: Use of the Clinical Frailty Scale for Critical Care Decisions. J Am Geriatr Soc. 2020;68(6):E30-2. https://dx.doi.org/10.1111/jgs.16528.

31. Owen RK, Conroy SP, Taub N, Jones W, Bryden D, Pareek M, et al. Comparing associations between frailty and mortality in hospitalised older adults with or without COVID-19 infection: a retrospective observational study using electronic health records. Age Ageing. 2020;afaa167. https://dx.doi.org/10.1093/ageing/afaa167.

32. Hewitt J, Carter B, Vilches-Moraga A, Quinn TJ, Braude P, Verduri A, et al. The effect of frailty on survival in patients with COVID19 (COPE): a multicentre, European, observational cohort study. Lancet Public Health. 2020;5(8):E444-51. https://doi.org/10.1016/ S2468-2667(20)30146-8.

33. Lapid MI, Koopmans R, Sampson EL, Van den Block L, Peisah C. Providing quality end of life care to older people in the era of COVID19: Perspectives from five countries. Int Psychogeriatr. 2020;1-8. https://dx.doi.org/10.1017/S1041610220000836.

34. Hubbard RE, Maier AB, Hilmer SN, Naganathans V, Etherton- Beer C, Rockwood K. Frailty in the Face of COVID-19. Age Ageing. 2020;49(4):499-500 https://dx.doi.org/10.1093/ageing/afaa095.

35. Felix, ZC, da Costa SF, Alves AM, de Andrade CG, Duarte MC, de Brito FM. Euthanasia, dysthanasia and orthothanasia: an integrative review of the literature. Cien Saude Colet. 2013;18(9):2733-46. https://dx.doi.org/10.1590/s1413-81232013000900029.

36. González C, Yama E, Yomayusa N, Vargas J, Rico J, Ariza A, et al. Consenso colombiano de expertos sobre recomendaciones informadas en la evidencia para la prevención, el diagnóstico y el manejo de la lesión renal aguda por SARS-CoV-2/COVID-19. Rev. Colomb. Nefrol. 2020;7(Suppl 2). https://doi.org/10.22265/acnef.7.Supl.2.473.

37. Vincent JL. Foregoing life support in western European intensive care units: the results of an ethical questionnaire. Crit Care Med. 1999;27(8):1626-33. https://dx.doi.org/10.1097/00003246-199908000-00042.

38. Monteiro F. Mechanical ventilation and medical futility or dysthanasia, the dialectic of high technology in intensive medicine. Rev Port Pneumol. 2006;12(3):281-91. https://dx.doi.org/10.1016/s0873-2159(15)30431-1.

39. Azulay-Tapiero A. Los principios bioéticos: ¿se aplican en la situación de enfermedad terminal? An Med Interna (Madrid). 2001;18(12):650-4 https://dx.doi.org/

40. Sahuquillo MR. 150.000 personas han registrado su testamento vital en España. El País. 20 de abril de 2013 [citado octubre 72020 ]. Disponible en: https://elpais.com/sociedad/2013/04/20/actualidad/1366478759_440365.html.

28 Voluntad anticipada versus distanasia en la atención del adulto mayor con COVID-19 y enfermedad renal 
Rev. Colomb. Nefrol. 2020;7(2):17-29, julio-diciembre de 2020 http://www.revistanefrologia.org

http://dx.doi.org/10.22265/acnef.7.2.519

41. Pi V. La mayor prueba de amor es que dejara de sufrir. Público. 20 de diciembre de 2010 [citado octubre 7 2020]. Disponible en: https:/ /www.publico.es/espana/mayor-prueba-amor-dejara-sufrir.html.

42. Reacción de DMD a la noticia de la muerte voluntaria de María José Carrasco con ayuda Ángel Hernández. DMD. 4 de abril de 2019 [citado octubre 7 2020]. Disponible en: https://derechoamorir.org/2019/04/04/reaccion-de-dmd-a-la-noticia-de-la-muerte-voluntariade-maria-jose-carrasco-con-ayuda-angel-hernandez/.

43. Ovidio González P1, Testimonios. DMD. Febrero 19 de 2018 [citado octubre 7 2020]. Disponible en: https://dmd.org.co/project/mi- papa-queria-buscar-a-la-muerte-con-la-frente-en-alto-matador/.

44. Meganoticias TVC. Voluntad anticipada para morir en paz Julio 20 de 2018 [citado octubre 7 2020]. Disponible en: https://www.youtube.com/watch?v=cbNT8rTlbjE.

45. Testimonio Eva Muciño/Voluntad Anticipada. Cáncer en positivo. Abril 7 de 2017. [citado octubre 7 2020]. Disponible en: https://www.youtube.com/watch?v=BZd99s1K1Us.

46. United Nations. Principios de las Naciones Unidas en favor de las personas de edad. United Nations; 1991 [citado octubre 6 2020]. Disponible en: https://www.un.org/development/desa/ageing/resources/international-year-of-older-persons-1999/principles/los-principiosde-las-naciones-unidas-en-favor-de-las-personas-de-edad.html.

47. Organización de Estados Americanos (OEA). Convención interamericana sobre la protección de los derechos humanos de las personas mayores (A-70). OEA; 2015. [citado octubre 6 2020]. Disponible en: http://www.oas.org/es/sla/ddi/tratados_multilaterales_interamericanos_a70_derechos_humanos_personas_mayores.asp\#: :text=El\%20objeto\%20de\%20la\%20Convención,y\%20participación\%20en\%20la\%20sociedad.

48. Colombia. Congreso de la República. Ley 23 de 1981 (febrero 18): Por la cual se dictan normas en materia de ética médica. Bogotá D.C.: Diario Oficial 35711; febrero 27 de 1981.

49. Colombia. Congreso de la república. Ley 1251 de 2008 (noviembre 27): Por la cual se dictan normas tendientes a procurar la protección, promoción y defensa de los derechos de los adultos mayores. Bogotá D.C.: Diario Oficial 47186; noviembre 27 de 2008.

50. Júdez J, Gracia D. La deliberación moral: el método de la ética clínica. Med Clin Barcelona. 2001;117(1):18-23. https://doi.org/10.1016/S0025-7753(01)71998-7.

51. León-Correa FJ. Las voluntades anticipadas: cómo conjugar autonomía y beneficencia. Análisis desde la bioética clínica. Rev CONAMED. 2008;13(3):26-32.

52. Colombia. Ministerio de Salud y Protección Social. Resolución 2665 de 2018 (junio 25): Por medio de la cual se reglamenta parcialmen- te la Ley 1733 de 2014 en cuanto al derecho a suscribir el documento de Voluntad Anticipada. Bogotá D.C.; junio 25 de 2018.

53. Bolívar-Góez PL, Gómez-Córdoba AI. Voluntades anticipadas al final de la vida. Una aproximación desde la regulación Colombiana y en el derecho comparado. Revista Latinoamericana de Bioética. 2016;16(1):128-53. http://dx.doi.org/10.18359/rlbi.1444.

54. Sánchez-Tomero JA, Rodríguez-Jornet A, Balda S, Cigarrán S, Herrero JC, Maduell F, et al. Evaluación de la opinión de los pacientes con enfermedad renal crónica en diálisis respecto al fin de la vida y la planificación anticipada de cuidados. Nefrología. 2011 [citado Septiembre 14 2020];31(4):449-56. Disponible en: http://scielo.isciii.es/scielo.php?script=sci_arttext\&pid=S0211-69952011000400009\&lng=es.

55. Rodríguez-Jornet A, Betancourt-Castellanos LA, Bolós-Contador MI, Oliva-Morera JC, Ibeas-López JA. Usefulness of questionnaires on advance directives in haemodialysis units. Nephrol Dial Transplant. 2017;32(10):1676-82. https://dx.doi.org/10.1093/ndt/gfx245.

56. Ssentongo P, Ssentongo AE, Heilbrunn ES, Ba DM, Chinchilli VM. Association of cardiovascular disease and 10 other pre-existing comorbidities with COVID-19 mortality: A systematic review and meta-analysis. PloS One. 2020;15(8):e0238215. https://doi.org/10.1371/journal.pone.0238215.

57. Roshanravan B, Patel KV. Assessment of physical functioning in the clinical care of the patient with advanced kidney disease. Semin Dial. 2019;32(4):351-60. https://doi.org/10.1111/sdi.12813.

58. Ruiz-Ospina PM. Retos de la geriatría en tiempos de Covid-19: toma de decisiones. Modelo pertinencia de atención en pacientes con enfermedad crónica disponible. En: Herramientas de cuidado paliativo, en tiempos de COVID-19, para no paliativistas. Santiago de Cali: Asociación Cuidados Paliativos de Colombia; 2020 [citado octubre 7 2020]. Disponible en: https://drive.google.com/file/d/19ubGn7YLXobNvxlMf1a_EEEgEanhdsSM/view. 